\title{
CoDesc: A Large Code-Description Parallel Dataset
}

\author{
Masum Hasan $^{1 *}$, Tanveer Muttaqueen ${ }^{1 *}$, Abdullah Al Ishtiaq ${ }^{1}$ \\ Kazi Sajeed Mehrab ${ }^{1}$, Md. Mahim Anjum Haque ${ }^{1}$, Tahmid Hasan ${ }^{1}$ \\ Wasi Uddin Ahmad ${ }^{2}$, Anindya Iqbal ${ }^{1}$, Rifat Shahriyar ${ }^{1}$ \\ ${ }^{1}$ Bangladesh University of Engineering and Technology (BUET) \\ ${ }^{2}$ University of California, Los Angeles \\ masumara.cse.buet.ac.bd, 1505002 .tmeugrad.cse.buet.ac.bd \\ rifatecse.buet.ac.bd
}

\begin{abstract}
Translation between natural language and source code can help software development by enabling developers to comprehend, ideate, search, and write computer programs in natural language. Despite growing interest from the industry and the research community, this task is often difficult due to the lack of large standard datasets suitable for training deep neural models, standard noise removal methods, and evaluation benchmarks. This leaves researchers to collect new small-scale datasets, resulting in inconsistencies across published works. In this study, we present CoDesc - a large parallel dataset composed of 4.2 million Java methods and natural language descriptions. With extensive analysis, we identify and remove prevailing noise patterns from the dataset. We demonstrate the proficiency of CoDesc in two complementary tasks for code-description pairs: code summarization and code search. We show that the dataset helps improve code search by up to $22 \%$ and achieves the new state-of-the-art in code summarization. Furthermore, we show CoDesc's effectiveness in pre-training-finetuning setup, opening possibilities in building pretrained language models for Java. To facilitate future research, we release the dataset, a data processing tool, and a benchmark at https://github.com/csebuetnlp/CoDesc.
\end{abstract}

\section{Introduction}

Neural models for natural language processing have benefited from large datasets and standard evaluation benchmarks (Wang et al., 2019b,a; Rajpurkar et al., 2016; Hermann et al., 2015; CommonCrawl). However, the programming language counterpart is lagging behind due to a lack in such large datasets and benchmarks. To put this into perspective, the original Transformer network

\footnotetext{
${ }^{*}$ Equal contribution.
}

(Vaswani et al., 2017) was trained on WMT'14 English-German and English-French datasets (Bojar et al., 2014) containing 4.5 million and 36 million parallel sentences, respectively, whereas a similar network that achieved state-of-the-art results in source code summarization has been trained on only 69 thousand code-description pairs (Ahmad et al., 2020). We argue that the existing models used for programming language tasks in the literature have a significant scope of improvement given a large, good-quality dataset, and such a dataset is the missing link for effectively applying deep learning methods on programming languages.

In this work, we collect and release a large (4.2 million) Java source code - natural language (NL) parallel dataset along with denoising methods and baseline results. We apply our dataset to established works in both training from scratch and pretraining-fine-tuning setting and we demonstrate a notable performance gain in both settings. We gain $10 \%$ to $22 \%$ improvement over baseline code search models using CoDesc, and attain performances comparable to models having $8 \times$ more parameters. We achieve a new state-of-the-art BLEU score of 45.89 in code summarization by pretraining a Transformer network with our dataset for two epochs. With extensive empirical analysis, we propose a set of noise removal techniques for the source code and the NL descriptions in our dataset.

Our work brings together several datasets and multiple tasks on the intersection of Natural Language Processing (NLP) and Software Engineering (SE), such as code summarization, code search and code synthesis, and allows researchers to compare their methods on the same benchmark. It also opens the door for building large pretrained models to jointly learn code and NL representations that can be leveraged in downstream tasks that do not have adequate data, such as, code refactoring, clone detection, etc. as done by Feng et al. (2020). 


\begin{tabular}{|c|c|c|c|c|c|c|c|c|c|}
\hline \multirow[b]{2}{*}{ Source } & \multirow[b]{2}{*}{ \#Projects } & \multirow[b]{2}{*}{$\begin{array}{c}\text { \#Raw } \\
\text { data }\end{array}$} & \multirow[b]{2}{*}{$\begin{array}{l}\text { \#Clean } \\
\text { data }\end{array}$} & \multicolumn{3}{|c|}{ Code } & \multicolumn{3}{|c|}{ Description } \\
\hline & & & & $\begin{array}{c}\text { \#Unique } \\
\text { tokens }\end{array}$ & $\begin{array}{l}\text { Avg } \\
\text { len }\end{array}$ & $\leq \mathbf{2 0 0}$ & $\begin{array}{c}\text { \#Unique } \\
\text { tokens }\end{array}$ & $\begin{array}{l}\text { Avg } \\
\text { len }\end{array}$ & $\leq \mathbf{5 0}$ \\
\hline CSN-Java & N/A & 542,991 & 490,169 & 284,214 & 140.41 & 83.42 & 168,507 & 25.14 & 89.42 \\
\hline DeepCom & 9,714 & 588,108 & 424,028 & 306,422 & 128.35 & 84.04 & 91,933 & 17.80 & 94.76 \\
\hline FunCom & 28,000 & $2,149,121$ & $2,130,247$ & 469,354 & 51.30 & 99.83 & 399,338 & 15.52 & 95.87 \\
\hline CONCODE & 33,000 & $2,184,310$ & 733,040 & 131,852 & 33.75 & 99.99 & 166,239 & 14.87 & 96.27 \\
\hline CSN-Py2Java & N/A & 456,000 & 434,032 & 414,018 & 163.78 & 72.32 & 223,277 & 57.11 & 68.69 \\
\hline CoDesc (All) & N/A & $5,920,530$ & $4,211,516$ & $1,128,909$ & 77.97 & 93.53 & 813,078 & 21.04 & 92.28 \\
\hline \multicolumn{10}{|c|}{ Balanced train-valid-test split for CoDesc data } \\
\hline train & - & 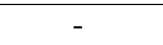 & $3,369,218$ & 991,395 & 78.01 & 93.53 & 718,204 & 21.05 & 92.28 \\
\hline valid & - & - & 421,149 & 269,435 & 77.73 & 93.51 & 188,145 & 21.08 & 92.26 \\
\hline test & - & - & 421,149 & 269,318 & 77.88 & 93.55 & 187,230 & 20.97 & 92.33 \\
\hline
\end{tabular}

Table 1: Statistics of CoDesc datasets and a balanced train-valid-test split. $\leq 200$ and $\leq 50$ indicates the percentage $(\%)$ of data where source code and description are smaller than 200 and 50 tokens, respectively.

\section{Related Works}

Code-Description Parallel Datasets With the advent of data-driven code search and code summarization methods, several datasets are proposed to facilitate research in code-NL parallel tasks. Husain et al. (2019) introduced CODESEARCHNET (CSN), a benchmark for code search techniques with 2.1 million code-NL parallel data in 6 programming languages, 6.4 million monolingual code data, a leader-board, and baseline results with 5 code search techniques. CONCODE (Iyer et al., 2018), DEEPCOM (Hu et al., 2018a), FunCom (LeClair and McMillan, 2019) are some notable dataset papers that released 2.18 million, 2.15 million, and 0.59 million parallel data respectively. Clement et al. (2020) released a parallel corpus of 26 million monolingual Python methods and 7.7 million method-docstring pairs. CoNaLa (Yin et al., 2018) is a Python line by line natural language description dataset containing nearly $3 \mathrm{k}$ parallel data.

\section{Code Search and Summarization CODE-NN} (Iyer et al., 2016) is a pioneering work in datadriven code summarization. The CodeSearchNet dataset paved the way for CodeBERT (Feng et al., 2020), a pretrained BERT (Devlin et al., 2019) model trained on CSN data with Masked Language Modeling (MLM) (Devlin et al., 2019) and Replaced Token Detection (RTD) (Clark et al., 2020) objective that achieved a high performance in the CSN benchmark. Wei et al. (2019) proposed a dual learning method that simultaneously trained code summarization and code generation and improved both of them using $60 \mathrm{k}$ parallel data. In the same dataset, Ahmad et al. (2020) achieved state-of-theart results in source code summarization using a
Transformer network (Vaswani et al., 2017). Along with the mentioned dataset, Clement et al. (2020) presented PyMT5, a text to text transformer that notably improved method generation and code summarization. Ahmad et al. (2021) collected more than 300 GB monolingual code and NL data, and trained PLBART, a pretrained seq2seq model for both program understanding and comprehension.

\section{CoDesc Dataset}

\subsection{Data Sources}

We collect our data from several sources and formulate rules for data cleaning. 5 of the authors spent 45-50 man-hours manually going over the dataset to identify patterns of noises in different data sources. Upon group discussion, common patterns were identified and a noise removal method was established. Details about these noise patterns are provided in Appendix A.

One of the datasets used in CoDesc is CODESEARCHNET $(\mathrm{CSN})^{1}$ (Husain et al., 2019) - a parallel method-description dataset for code search. Furthermore, other datasets used are DeepCom ${ }^{2}$ (Hu et al., 2018a), CONCODE$^{3}$ (Iyer et al., 2018), FunCom ${ }^{4}$ (LeClair and McMillan, 2019) - datasets created for code summarization. The CODESEARCHNET dataset originally contained 6 programming languages, from which the Java methods are directly used in CoDesc, however, the Python methods are used after being automatically translated to Java. We combine all aforementioned datasets to create CoDesc. Appendix B shows a

\footnotetext{
${ }^{1}$ https://github.com/github/CodeSearchNet

${ }^{2}$ https://github.com/xing-hu/DeepCom

${ }^{3}$ https://github.com/sriniiyer/concode

${ }^{4} \mathrm{http} / / /$ leclair.tech/data/funcom/
} 
sample code-description parallel data from each of these datasets. Table 1 describes our data sources and their characteristics in detail.

CSN Python to Java Translation To utilize maximum possible data from the CSN CORPUS, we translate the Python methods to Java using TransCoder (Lachaux et al., 2020), a state-of-theart, neural source-to-source compiler. We modified and re-released the open-source implementation of TransCoder ${ }^{5}$, enabling it to translate data in batches instead of one at a time, and resulting in a 16X faster translation. Upon empirical inspection, we found that the converted Java codes are human-readable and bear a strong resemblance to the original Python code intent. The converted codes seem correct to the human eye and their syntax matches with Java syntax. However, a few cases the transcompiler suffers are - converting to Java library methods, and converting from Python coding conventions that does not have a Java equivalent (e.g. use of SELF). These conversion errors, however, were not severe enough to affect our model to learn the NL-source code mapping.

\subsection{Data Cleaning and Noise Removal}

We created an easy-to-use, parameterized data processing tool for removing the different types of noise that we observed in our dataset. From the natural language descriptions, we remove symbols and characters that do not carry a meaning in a natural language description, such as, comment tags (e.g., //, /*, */), stray code characters (e.g., e, \#, $\{$,$\} , etc.), HTML and XML tags, non-$ ASCII and escape characters, and some patterns of autogenerated tags (e.g., Aparam, @return, ethrows, etc.). From source code, we remove comments and the non-ASCII and escape characters. In previous studies, many meaningful data are discarded due to having some noisy patterns/symbols either in the code or description (Husain et al., 2019; Iyer et al., 2018; LeClair and McMillan, 2019). We identify and remove the noisy part of the data points without excluding them from the dataset to reduce data loss during preprocessing.

For both source code and NL description, we split CamelCase and snake_case code tokens into subtokens (e.g., Camel Case, snake case) and separate linked alphabets and numbers (e.g., var0 to var 0) (Ahmad et al., 2020; LeClair and McMillan,

\footnotetext{
${ }^{5}$ https://github.com/csebuetnlp/TransCoder
}

2019). After the aforementioned processing, we remove the data points where the source code is less than 3 tokens, or the description contains less than 2 alphabets (Husain et al., 2019). We lowercase the natural language as the case is not necessary for describing codes. We release our data processing tool along with the CoDesc dataset for applying the dataset to diverse tasks.

\subsection{Dataset Characteristics}

After the previous steps, we are left with nearly 4.2 million Java method and description parallel data. Table 1 presents the statistics characteristics of our dataset. The combined CoDesc dataset consists of more than one million unique tokens, which is significantly larger than natural language vocabulary (Chen et al., 2019). This can be partially attributed to inseparable multi-words (e.g. 'updateproductvariationlocalizeddeltaprice') in our dataset. Hence, we perform BPE (Sennrich et al., 2016) tokenization in our preprocessing pipeline. We also see that although the average token length of Java source codes vary in the different dataset sources, the natural language descriptions have a relatively uniform length. We create a balanced, deduplicated, and representative train-valid-test dataset by splitting individual source-dataset in 8:1:1 ratio (Table 1).

\section{Experiments}

We evaluate our code-description corpus in two well-known complementary tasks: source code summarization and natural language code search. In this section, we demonstrate that models trained on CoDesc bring about a noticeable improvement over two established baselines in code search and code summarization. Each benchmarking follows a standard cleaning, preprocessing, and train-test de-duplication process.

\subsection{Natural Language Code Search}

We use the code search models used by Husain et al. (2019) that jointly trains a source code and an NL encoder networks to minimize their encoded vector distance (Figure. 1). We apply our dataset on the CodeSEARChNet (CSN) (Husain et al., 2019) - a well-studied benchmark in the semantic code search literature. We train 5 different encoder networks (Table. 2) with the CSN Java dataset, and CoDesc respectively. We compare our results with CodeBERT and RoBERTa (code) (Feng et al., 2020), two pretrained models achieving state-of- 


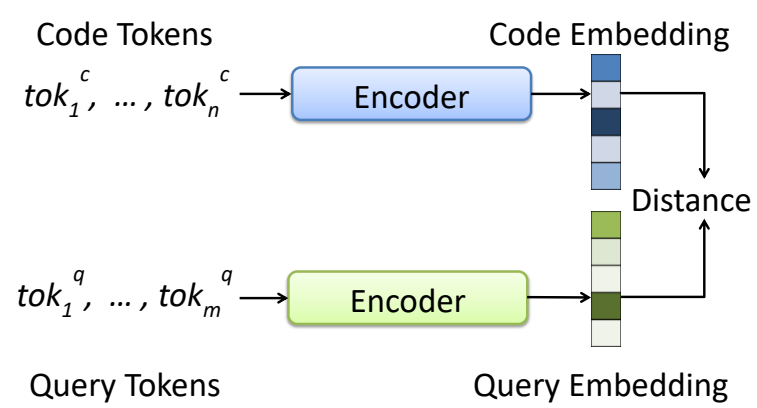

Figure 1: Code search model architecture; code and NL (query) encoders jointly train to reduce their embedded distance. During search, we select the code that is closest to the query in their shared embedding space.

\begin{tabular}{l|c|c|c}
\hline \multirow{2}{*}{ Model } & \multirow{2}{*}{ \#Param } & \multicolumn{2}{c}{ CSN test MRR } \\
\cline { 3 - 4 } & & CSN-Java & CoDesc \\
\hline NBOW & $11.6 \mathrm{M}$ & 0.589 & 0.683 \\
RNN & $12.6 \mathrm{M}$ & 0.582 & 0.679 \\
Sel-attn & $13.6 \mathrm{M}$ & 0.583 & 0.723 \\
1D Conv & $16.4 \mathrm{M}$ & 0.520 & 0.686 \\
Conv self-attn & $16.0 \mathrm{M}$ & 0.509 & $\mathbf{0 . 7 2 9}$ \\
\hline State-of-the-art models & \multicolumn{3}{|c}{0.721} \\
\hline RoBERTa (code) & $125 \mathrm{M}$ & $\mathbf{0 . 7 4 8}$ \\
\hline
\end{tabular}

Table 2: Baseline models trained with default dataset and CoDesc, along with, comparison with SoTA pretrained models in CSN Java test set. Training on CoDesc outperforms training on CSN-Java only, and it is comparable to SOTA with $8 \mathrm{x}$ fewer parameters.

the-art results in CSN Benchmark. They are trained with a Masked Language Modeling (MLM) (Devlin et al., 2019) objective on 2.1 million bimodal code-NL data, and 6.4 million unimodal data released with CODESEARCHNET.

Results We use Mean Reciprocal Rank (MRR) - the commonly used evaluation metric for code search (Husain et al., 2019; Sachdev et al., 2018; Cambronero et al., 2019) as the evaluation criteria for code search. Table 2 shows our results, along with state-of-the-art models (Liu et al., 2019; Feng et al., 2020) that have nearly 8-10 times more parameters than the baseline networks and a more complex training objective. We achieve remarkably close performance with the state-of-the-art models with much simpler and smaller networks.

\subsection{Source Code Summarization}

For this task, we follow the methodology proposed by Ahmad et al. (2020). They used a seq2seq Transformer (Vaswani et al., 2017) network with 77M parameters with relative positional encoding (Shaw

\begin{tabular}{l|ccc}
\hline Methods & BLEU & METEOR & ROUGE-L \\
\hline Transformer & 44.58 & 26.43 & 54.76 \\
CoDesc pretrained & $\mathbf{4 5 . 8 9}$ & $\mathbf{2 8 . 0 1}$ & $\mathbf{5 6 . 5 9}$ \\
\hline
\end{tabular}

Table 3: Code summarization with Ahmad et al. (2020) proposed Transformer network with and without pretraining with CoDesc.

et al., 2018) and copying mechanism (See et al., 2017) and achieved state-of-the-art results.

Data preparation Ahmad et al. (2020) used a Java dataset released by Hu et al. (2018b) and preprocessed by Wei et al. (2019) consisting of training, validation, and test datasets of size 69,708, 8,714 , and 8,714 respectively. We refer to this training data as train-small. We create a new dataset CoDesc-train by combining train-small with CoDesc. We replace all literals as Wei et al. (2019) and tokenize the dataset using Character BPE Tokenization (Sennrich et al., 2016) to create the same size vocabulary as the previous works.

Training We train a Transformer model proposed by Ahmad et al. (2020) with CoDesc-train dataset. We use Adam optimizer with an initial learning rate of $10^{-4}$, mini-batch size of 32 , and dropout rate 0.2 , vocabulary size $50 \mathrm{k}$ for code and $30 \mathrm{k}$ for NL. However, we use maximum input length of 200 token instead of 150 based on our observation of CoDesc dataset from Table 1. Each epoch of the model took nearly 8 hours in an NVIDIA V100 16GB GPU. In comparison, the train-small dataset took 8.5 minutes only. For limitation of computational resource, we saved the network weights after training it with the large dataset for two epochs, and to be consistent with the original implementation, trained them further with the train-small dataset for a maximum of 198 more epochs. We perform an early stop if the validation performance does not improve for consecutive 20 epoch. The pretraining provides the network parameters a more favorable initialization than random, helping the network find better local minima.

Results Table 3 shows that our two epoch pretraining with CoDesc significantly improves the state-of-the-art code summarization methods in all three evaluation metrics - BLEU (Papineni et al., 2002), METEOR (Banerjee and Lavie, 2005), and ROUGE-L (Lin, 2004). We observe that the pretrained model often generates more descriptive summary even when it achieves lower BLEU score (Fig. 2). We believe the model has more room for 
public void makeImmutable() \{

if (mutable)

if (results ! = null)

int length = results.size();

for (int $i=\mathrm{NUM} ; i<$ length; $i++$ )

Result result $=$ (Result)results.get(i); result.makeImmutable ();

\} results $=$ Collections.unmodifiableList (results);

\} mutable = BOOL; \}

Human written: makes the object immutable

Transformer prediction (BLEU: 1.0): makes the object immutable

CoDesc pretrained model prediction (BLEU: 0.12): if there are any object in the list then the object is not immutable

Figure 2: CoDesc pretrained model generates more descriptive summary, even in cases it achieves lower score.

\begin{tabular}{l|ccr}
\hline Dataset & $\begin{array}{c}\text { Raw } \\
\text { data }\end{array}$ & $\begin{array}{c}\text { Clean } \\
\text { data }\end{array}$ & Inc. (\%) \\
\hline CSN (Java) & 0.5870 & 0.6427 & 5.57 \\
DeepCom & 0.4677 & 0.6069 & 13.92 \\
FunCom & 0.5379 & 0.6366 & 9.87 \\
CONCODE & 0.5444 & 0.6234 & 7.90 \\
CSN (Python2Java) & 0.5081 & 0.5546 & 4.65 \\
CoDesc (All) & 0.5852 & 0.6826 & 9.74 \\
\hline
\end{tabular}

Table 4: MRR of individual datasets (Section 3.1) before and after noise removal.

improvement with further pretraining and we wish to validate this in future work.

\subsection{Ablation \& Analysis}

To quantify the effect of individual data sources and our noise removal methodology, we train each dataset before and after applying our data cleaning method using an NBOW model and test them in the CSN benchmark using their released test set.

Although our collected data was already cleaned by the respective authors, Table 4 shows that the performance of every dataset improves drastically after our noise removal. Interestingly, without our extra layer of data cleaning, CoDesc dataset performs worse than training with only CSN data although being significantly larger. This shows the importance of a standard cleaning and processing method. Moreover, CSN (Java) have the highest accuracy, which can be attributed to the fact that it came from the same distribution of data as the evaluation and test sets, and hence contains similar tokens and patterns (Husain et al., 2019). We can see from Table 4 that the model trained with CSN (Python2Java) achieves an MRR score of 0.5548 . Although this score is lower than other datasets, it is still a good indication that the translated data is helping the model is to learn NL-code association.

New Benchmark Results in Code Search We provide a new set of benchmark results for CoDesc dataset in natural language code search. We train, validate, and test an NBOW, an RNN, and a Selfattn code search network with the balanced train, validation, and test data shown in Table 1. The three models achieve MRR score of $\mathbf{0 . 8 1 2}, \mathbf{0 . 7 6 6}$, and $\mathbf{0 . 8 3 9}$ respectively.

\section{Discussion and Conclusion}

In this work, we have accumulated CoDesc - a large code-description parallel dataset and established baseline results. CoDesc brings a noteworthy improvement in two tasks: code search and code summarization. We believe CoDesc will serve as a base for future studies on code-description joint tasks. We also show that automatically translated source code from a source-to-source compiler can be applied in a code-NL parallel task, suggesting that, translating our Java dataset to other programming languages can also be helpful.

The most striking finding of our study is that, by training with $2 \mathrm{X}$ larger parallel data, we achieve equivalent performance to models having $8 \mathrm{X}$ parameters (Feng et al., 2020) in code search. This raises an interesting question: are we fully utilizing the model capacities in code-description studies? From our pretraining results in code summarization, it can be reasonably assumed that pretraining with our large dataset the larger models will also improve further. In future works, we wish to apply new techniques for code search, code summarization, along with exploring our dataset for generalpurpose code synthesis, where the best models are still struggling in accuracy (Wei et al., 2019; Yin and Neubig, 2017).

\section{Acknowledgement}

We thank the ICT Division, Bangladesh for funding the project and Intelligent Machines Limited for providing the cloud support. 


\section{References}

Wasi Ahmad, Saikat Chakraborty, Baishakhi Ray, and Kai-Wei Chang. 2020. A transformer-based approach for source code summarization. In Proceedings of the 58th Annual Meeting of the Association for Computational Linguistics, pages 4998-5007, Online. Association for Computational Linguistics.

Wasi Ahmad, Saikat Chakraborty, Baishakhi Ray, and Kai-Wei Chang. 2021. Unified pre-training for program understanding and generation. In Proceedings of the 2021 Conference of the North American Chapter of the Association for Computational Linguistics: Human Language Technologies, pages 2655-2668, Online. Association for Computational Linguistics.

Satanjeev Banerjee and Alon Lavie. 2005. METEOR: An automatic metric for MT evaluation with improved correlation with human judgments. In Proceedings of the ACL Workshop on Intrinsic and Extrinsic Evaluation Measures for Machine Translation and/or Summarization, pages 65-72, Ann Arbor, Michigan. Association for Computational Linguistics.

Ondřej Bojar, Christian Buck, Christian Federmann, Barry Haddow, Philipp Koehn, Johannes Leveling, Christof Monz, Pavel Pecina, Matt Post, Herve Saint-Amand, Radu Soricut, Lucia Specia, and Aleš Tamchyna. 2014. Findings of the 2014 workshop on statistical machine translation. In Proceedings of the Ninth Workshop on Statistical Machine Translation, pages 12-58, Baltimore, Maryland, USA. Association for Computational Linguistics.

Jose Cambronero, Hongyu Li, Seohyun Kim, Koushik Sen, and Satish Chandra. 2019. When deep learning met code search. In Proceedings of the 2019 27th ACM Joint Meeting on European Software Engineering Conference and Symposium on the Foundations of Software Engineering, ESEC/FSE 2019, pages 964-974.

Wenhu Chen, Yu Su, Yilin Shen, Zhiyu Chen, Xifeng Yan, and William Yang Wang. 2019. How large a vocabulary does text classification need? a variational approach to vocabulary selection. In Proceedings of the 2019 Conference of the North American Chapter of the Association for Computational Linguistics: Human Language Technologies, Volume 1 (Long and Short Papers), pages 3487-3497, Minneapolis, Minnesota. Association for Computational Linguistics.

Kevin Clark, Minh-Thang Luong, Quoc V. Le, and Christopher D. Manning. 2020. ELECTRA: Pretraining text encoders as discriminators rather than generators. In ICLR.

Colin Clement, Dawn Drain, Jonathan Timcheck, Alexey Svyatkovskiy, and Neel Sundaresan. 2020. PyMT5: multi-mode translation of natural language and python code with transformers. In Proceed ings of the 2020 Conference on Empirical Methods in Natural Language Processing (EMNLP), pages 9052-9065, Online. Association for Computational Linguistics.

CommonCrawl. Common crawl. https:// commoncrawl.org/. Accessed: 2021-01-31.

Jacob Devlin, Ming-Wei Chang, Kenton Lee, and Kristina Toutanova. 2019. BERT: Pre-training of deep bidirectional transformers for language understanding. In Proceedings of the 2019 Conference of the North American Chapter of the Association for Computational Linguistics: Human Language Technologies, Volume 1 (Long and Short Papers), pages 4171-4186, Minneapolis, Minnesota. Association for Computational Linguistics.

Zhangyin Feng, Daya Guo, Duyu Tang, Nan Duan, Xiaocheng Feng, Ming Gong, Linjun Shou, Bing Qin, Ting Liu, Daxin Jiang, and Ming Zhou. 2020. CodeBERT: A pre-trained model for programming and natural languages. In Findings of the Association for Computational Linguistics: EMNLP 2020, pages 1536-1547, Online. Association for Computational Linguistics.

Karl Moritz Hermann, Tomáš Kočiský, Edward Grefenstette, Lasse Espeholt, Will Kay, Mustafa Suleyman, and Phil Blunsom. 2015. Teaching machines to read and comprehend. In Proceedings of the 28th International Conference on Neural Information Processing Systems (NIPS 2015), pages 1693-1701, Montreal, Canada.

Xing Hu, Ge Li, Xin Xia, David Lo, and Zhi Jin. 2018a. Deep code comment generation. In Proceedings of the 26th Conference on Program Comprehension, ICPC' 18, page 200-210.

Xing Hu, Ge Li, Xin Xia, David Lo, Shuai Lu, and Zhi Jin. 2018b. Summarizing source code with transferred api knowledge. In Proceedings of the TwentySeventh International Joint Conference on Artificial Intelligence, IJCAI-18, pages 2269-2275. International Joint Conferences on Artificial Intelligence Organization.

Hamel Husain, Ho-Hsiang Wu, Tiferet Gazit, Miltiadis Allamanis, and Marc Brockschmidt. 2019. Codesearchnet challenge: Evaluating the state of semantic code search. arXiv preprint arXiv:1909.09436.

Srinivasan Iyer, Ioannis Konstas, Alvin Cheung, and Luke Zettlemoyer. 2016. Summarizing source code using a neural attention model. In Proceedings of the 54th Annual Meeting of the Association for Computational Linguistics (Volume 1: Long Papers), pages 2073-2083, Berlin, Germany. Association for Computational Linguistics.

Srinivasan Iyer, Ioannis Konstas, Alvin Cheung, and Luke Zettlemoyer. 2018. Mapping language to code in programmatic context. In Proceedings of the 2018 Conference on Empirical Methods in Natural Language Processing, pages 1643-1652, Brussels, Belgium. Association for Computational Linguistics. 
Marie-Anne Lachaux, Baptiste Roziere, Lowik Chanussot, and Guillaume Lample. 2020. Unsupervised translation of programming languages. In Proceedings of the 34th International Conference on Neural Information Processing Systems (NeurIPS 2020).

Alexander LeClair and Collin McMillan. 2019. Recommendations for datasets for source code summarization. In Proceedings of the 2019 Conference of the North American Chapter of the Association for Computational Linguistics: Human Language Technologies, Volume 1 (Long and Short Papers), pages 3931-3937, Minneapolis, Minnesota. Association for Computational Linguistics.

Chin-Yew Lin. 2004. ROUGE: A package for automatic evaluation of summaries. In Text Summarization Branches Out, pages 74-81, Barcelona, Spain. Association for Computational Linguistics.

Yinhan Liu, Myle Ott, Naman Goyal, Jingfei Du, Mandar Joshi, Danqi Chen, Omer Levy, Mike Lewis, Luke Zettlemoyer, and Veselin Stoyanov. 2019. Roberta: A robustly optimized bert pretraining approach. arXiv preprint arXiv:1907.11692.

C. Lopes, S. Bajracharya, J. Ossher, and P. Baldi. 2010. UCI source code data sets.

Kishore Papineni, Salim Roukos, Todd Ward, and WeiJing Zhu. 2002. Bleu: a method for automatic evaluation of machine translation. In Proceedings of the 40th Annual Meeting of the Association for Computational Linguistics, pages 311-318, Philadelphia, Pennsylvania, USA. Association for Computational Linguistics.

Pranav Rajpurkar, Jian Zhang, Konstantin Lopyrev, and Percy Liang. 2016. SQuAD: 100,000+ questions for machine comprehension of text. In Proceedings of the 2016 Conference on Empirical Methods in Natural Language Processing, pages 2383-2392, Austin, Texas. Association for Computational Linguistics.

Saksham Sachdev, Hongyu Li, Sifei Luan, Seohyun Kim, Koushik Sen, and Satish Chandra. 2018. Retrieval on source code: a neural code search. In Proceedings of the 2nd ACM SIGPLAN International Workshop on Machine Learning and Programming Languages, MAPL 2018, page 31-41.

Abigail See, Peter J. Liu, and Christopher D. Manning. 2017. Get to the point: Summarization with pointergenerator networks. In Proceedings of the 55th Annual Meeting of the Association for Computational Linguistics (Volume 1: Long Papers), pages 10731083, Vancouver, Canada. Association for Computational Linguistics.

Rico Sennrich, Barry Haddow, and Alexandra Birch. 2016. Neural machine translation of rare words with subword units. In Proceedings of the 54th Annual Meeting of the Association for Computational
Linguistics (Volume 1: Long Papers), pages 17151725, Berlin, Germany. Association for Computational Linguistics.

Peter Shaw, Jakob Uszkoreit, and Ashish Vaswani. 2018. Self-attention with relative position representations. In Proceedings of the 2018 Conference of the North American Chapter of the Association for Computational Linguistics: Human Language Technologies, Volume 2 (Short Papers), pages 464-468, New Orleans, Louisiana. Association for Computational Linguistics.

Ashish Vaswani, Noam Shazeer, Niki Parmar, Jakob Uszkoreit, Llion Jones, Aidan N Gomez, Łukasz Kaiser, and Illia Polosukhin. 2017. Attention is all you need. In Proceedings of the 31st International Conference on Neural Information Processing Systems (NIPS 2017), page 6000-6010, Long Beach, California, USA.

Alex Wang, Yada Pruksachatkun, Nikita Nangia, Amanpreet Singh, Julian Michael, Felix Hill, Omer Levy, and Samuel Bowman. 2019a. SuperGLUE: A stickier benchmark for general-purpose language understanding systems. In Proceedings of the 33rd International Conference on Neural Information Processing Systems (NeurIPS 2019), pages 3266-3280, Vancouver, Canada.

Alex Wang, Amanpreet Singh, Julian Michael, Felix Hill, Omer Levy, and Samuel R. Bowman. 2019b. GLUE: A multi-task benchmark and analysis platform for natural language understanding. In $7 \mathrm{th}$ International Conference on Learning Representations, ICLR 2019, New Orleans, LA, USA, May 6-9, 2019. OpenReview.net.

Bolin Wei, Ge Li, Xin Xia, Zhiyi Fu, and Zhi Jin. 2019. Code generation as a dual task of code summarization. In Proceedings of the 33rd International Conference on Neural Information Processing Systems (NeurIPS 2019), pages 6563-6573, Vancouver, Canada.

Pengcheng Yin, Bowen Deng, Edgar Chen, Bogdan Vasilescu, and Graham Neubig. 2018. Learning to mine aligned code and natural language pairs from stack overflow. In International Conference on Mining Software Repositories, MSR, pages 476-486. ACM.

Pengcheng Yin and Graham Neubig. 2017. A syntactic neural model for general-purpose code generation. In Proceedings of the 55th Annual Meeting of the Association for Computational Linguistics (Volume 1: Long Papers), pages 440-450, Vancouver, Canada. Association for Computational Linguistics. 


\section{Supplementary Material: Appendices}

\section{A Dataset Details}

CodeSearchNet (CSN) Corpus is a code search dataset for 6 programming languages (Husain et al., 2019). ${ }^{6}$ Despite the authors' effort for data cleaning, in our observation, CSN CORPUS is one of the noisiest. The dataset contains duplicate descriptions, inseparable multi-words (e.g., updateproductvariationlocalizeddeltaprice, updatelocationinventory), XML tags (e.g., <tt>, <soup>, <sub>), non-English documentation, non-ASCII and escape characters, unwanted symbols (e.g., @, \#, $\{$,$\} ), deprecated methods and descriptions,$ comments inside code, annotations (e.g., @ link, (code, ainheritdoc) in description, etc. Datapoints truncated by TransCoder during Python to Java translation (total 27,471) are marked with a special flag in our released corpus.

DeepCom Hu et al. (2018a) released a dataset of 588,108 Java method and documentation pairs collected from 9,714 GitHub projects for code summarization $^{7}$. Similar to CODESEARCHNET (Husain et al., 2019), they considered the first sentence of a documentation as the summary of the method as it typically describes the functionalities of Java methods. They filter out empty and single world descriptions and the setter, getter, constructors, and test methods, since they are easy for a model to summarize. In our manual analysis, we found HTML tags (e.g. $\langle t t\rangle,\langle\mathrm{p}\rangle,\langle\mathrm{p}$ class $=$ $\ldots\rangle,\langle l i\rangle,\langle u l\rangle)$, comment tags, annotations, escape characters inside descriptions, empty parentheses as descriptions, repetitive and nonmeaningful descriptions, comments inside source code, etc. Despite the authors' claim, we found numerous test methods in the dataset, which were mostly meaningful data.

CONCODE Iyer et al. (2018) released a dataset named CONCODE, collected by mining nearly 33,00 GitHub repositories ${ }^{8}$. In their preprocessed dataset, they replaced the names of the identifier and method names with generic terms, (e.g., $\arg 0$, loc0, function, etc.) and replaced all string literals with constants. This created a discrepancy with the other datasets, hence, we opted for their

\footnotetext{
${ }^{6}$ https://github.com/github/CodeSearchNet

${ }^{7}$ https://github.com/xing-hu/DeepCom

${ }^{8} \mathrm{https}: / /$ github.com/sriniiyer/concode
}

unprocessed dataset rather than the processed version. The unprocessed dataset released with $\mathrm{CON}$ CODE contained approximately 2.1 million Java methods and lowercased Javadoc-style document pairs. Upon duplicate removal, we were left with 733,878 datapoints.

Although some noises were present in this dataset, we found this data to be least noisy in manual observation. We find that because of lower casing the documentations, some CamelCase tokens became inseparable. The dataset also contained non-English comments with English alphabets (mostly Italian). We found these documents hard to identify and remove.

FunCom LeClair and McMillan (2019) released a dataset of over 2.1 million pairs of Java methods and one-sentence method descriptions from over $28 \mathrm{k}$ Java projects ${ }^{9}$. They collected this dataset by filtering over 51 million Java methods from UCI Source Code datasets (Lopes et al., 2010). In their preprocessing step, LeClair and McMillan (2019) removed all datapoints where the method is more than 100 tokens long, or the method description is over 13 tokens or below 3 tokens.

In our observation of this dataset, we found method descriptions containing HTML tokens (e.g. <tt>, annotations (e.g., alink, @param), comment tokens, unwanted symbols, solely nonalphabetic characters, etc. It also contained comments inside methods, and a large portion of the data were getter, setter, tester, and tostring methods.

\section{B Sample Data}

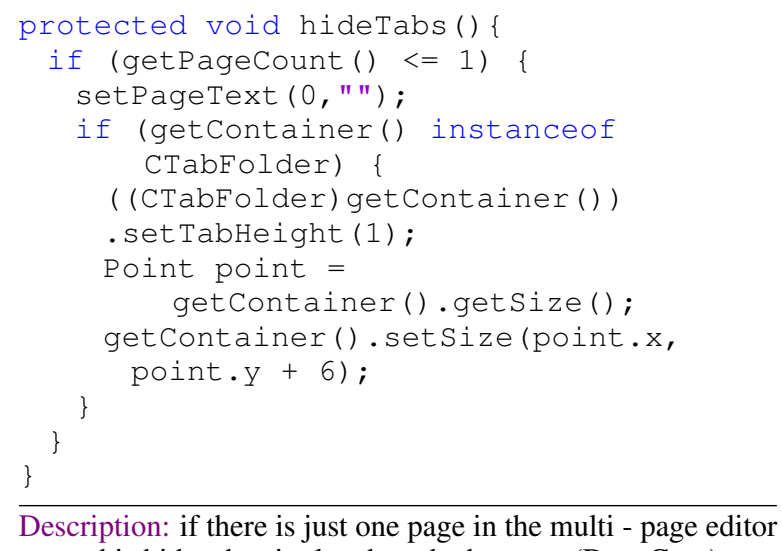

Description: if there is just one page in the multi - page editor part , this hides the single tab at the bottom. (DeepCom)

${ }^{9}$ http://leclair.tech/data/funcom/ 


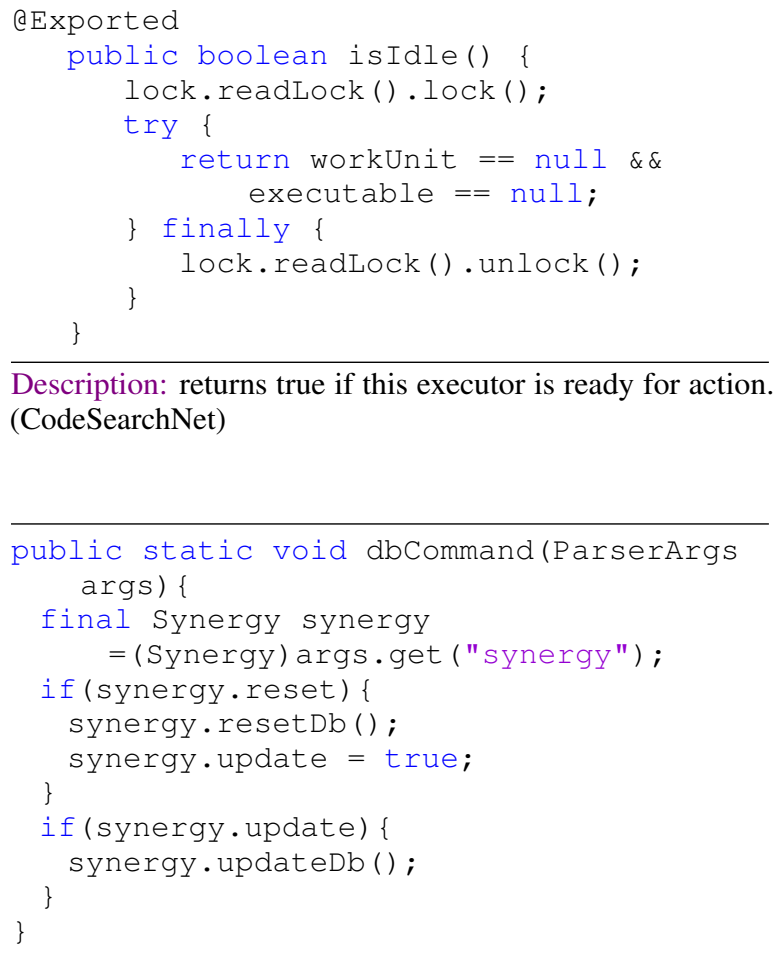

Description: returns true if this executor is ready for action. (CodeSearchNet)

Description: manages synergy db state (CodeSearchNetPython to Java)

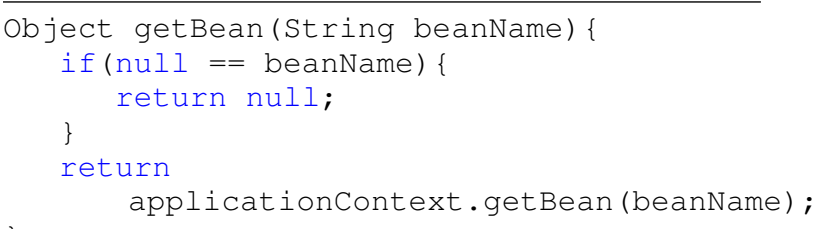
note that this may result in new bean creation if the scope is set to "prototype" in the bean configuration file. (CONCODE)

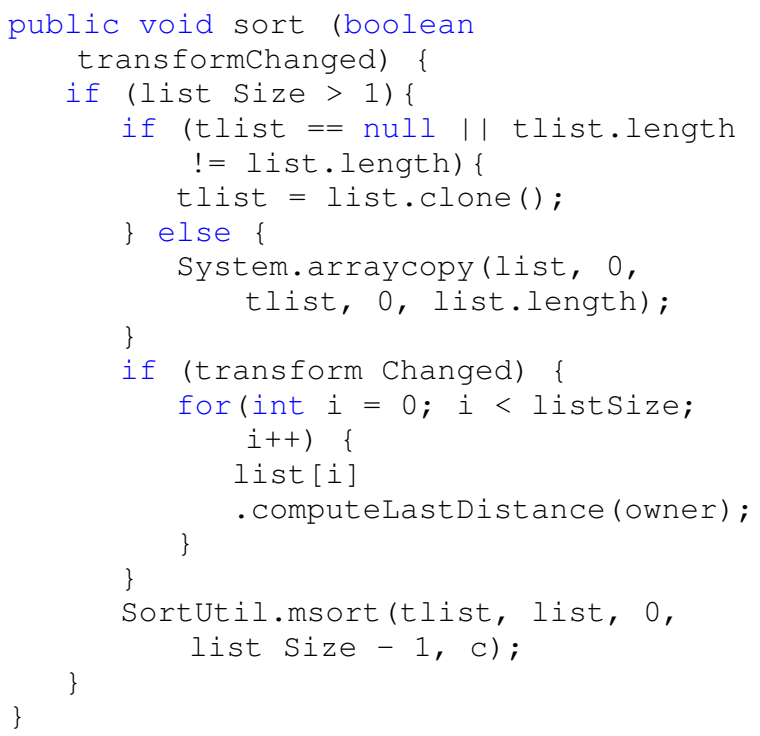

Description: sorts the elements in the list acording to their comparator. there are two reasons why lights should be resorted. first, if the lights have moved, that means their 